\title{
Vibrational spectra of pilocarpine hydrochloride crystals
}

\author{
R.R.F. Bento \\ Instituto de Física, Universidade Federal do Mato Grosso, Cuiabá-MT, Brazil \\ P.T.C. Freire* \\ Departamento de Física, Universidade Federal do Ceará, Fortaleza-CE, Brazil
}

A.M.R. Teixeira and J.H. Silva

Dep. Ciências Físicas e Biológicas, Universidade Regional do Cariri, Crato-CE, Brazil

J.A. Lima Jr.

Universidade Estadual do Ceará, Limoeiro do Norte-CE, Brazil

M.C.F. de Oliveira and M. Andrade-Neto

Dep. Química Orgânica e Inorgânica, Universidade Federal do Ceará, Fortaleza-CE, Brazil

N.R. Romero

Departamento de Farmácia, Universidade Federal do Ceará, Fortaleza-CE, Brazil

F.M. Pontes

Faculdade de Ciências, Universidade Estadual de São Paulo, Bauru-SP, Brasil

(Received on 15 November, 2008)

\begin{abstract}
Pilocarpine is a natural substance with potential application in the treatment of several diseases. In this work Fourier Transform (FT)-Raman spectrum and the Fourier Transform infra red (FT-IR) spectrum of pilocarpine hydrochloride $\mathrm{C}_{11} \mathrm{H}_{17} \mathrm{~N}_{2} \mathrm{O}_{2}^{+}$. $\mathrm{Cl}^{-}$were investigated at $300 \mathrm{~K}$. Vibrational wavenumber and wave vector have been predicted using density functional theory (B3LYP) calculations with the 6-31 G(d,p) basis set. A comparison with experiment allowed us to assign most of the normal modes of the crystal.
\end{abstract}

Keywords: Raman scattering, infrared spectroscopy, normal modes, pilocarpine hydrochloride

\section{INTRODUCTION}

In recent years, there has been a growing interest in the study of spectroscopic properties of plant cells in order to identify their chemical constituents through non-destructive analysis [1]. The main researches deal with primary metabolites, i.e., substances essential for their growth, surviving and reproduction (among them, amino acids, proteins, carbohydrates, lipids and fatty acids). On the contrary, the investigation of the vibrational property of isolated secondary metabolites from plants (used as defense against parasite and diseases as well as used to reinforce reproductive processes) is still poorly explored, although many of them have potential application as therapeutic drugs $[2,3]$.

Secondary metabolity pilocarpine $\left(\mathrm{C}_{11} \mathrm{H}_{17} \mathrm{~N}_{2} \mathrm{O}_{2}\right)$, an alkaloid extracted from the leaves of the South American shrubs Pilocarpus jaborandi, Pilocarpus microphyllus and other Pilocarpus species [4], is an imidazole derivative that exhibits some pharmacological activities. These activities include diaphoretic effects, stimulation of parasympathetic system [5], miotic action [6], being also used in ophthalmology [6,7]. The action of pilocarpine on the parasymphatetic nervous system has been extensively investigated and it is known that the substance act mainly as a cholinergic drug [8]. Despite of several therapeutic effects, pilocarpine is used clinically only to treat

*Electronic address: tarso@fisica.ufc.br glaucoma [8].

Pilocarpine molecule, which contains both imidazole and $\gamma$ - lactone rings forms two semi-organic compounds in the solid state phase, trichlorogermanate hermihydrate [9] $\left(\mathrm{C}_{11} \mathrm{H}_{17} \mathrm{~N}_{2} \mathrm{O}_{2}, \mathrm{GeCl}_{3}, 1 / 2 \mathrm{H}_{2} \mathrm{O}\right)$ and hydrochloride $\left(\mathrm{C}_{11} \mathrm{H}_{17} \mathrm{~N}_{2} \mathrm{O}_{2}^{+} . \mathrm{Cl}^{-}\right)$. [10] For both compounds it was discovered that the crystal structures are monoclinic, space group $P 2{ }_{1}$, although the conformation of the pilocarpine molecule itself differs significantly from one structure to the other $[9,10]$.

From the biological point of view, pilocarpine hydrochloride has been used in certain eye diseases, as for exemple, in the treatment of intraocular hemorrhages, opacities of the vitreous and aqueous fluids [8], while trichlorogermanate hermihydrate pilocarpine presents a weak activity of muscarinic stimulants [11].

In this work an infrared analysis and a Raman scattering study in the spectral range $40 \mathrm{~cm}^{-1}$ to $4000 \mathrm{~cm}^{-1}$ of pilocarpine hydrochloride crystal obtained from Pilocarpus trachyllophys [4] is reported. In order to assign the normal modes of vibrations of the material a Density Functional Theory (DFT) calculation was performed.

\section{EXPERIMENTAL}

FT-Raman spectrum was taken using a Bruker RFS100/S FTR system and a D418-T detector, with the sample excited by means of the $1064 \mathrm{~nm}$ line of a Nd:YAG laser. Infrared spectrum was obtained by using an Equinox/55 (Bruker) Fourier Transformed Infrared (FTIR) spectrometer. FT-Raman and 
FT-IR spectra were collected from samples confined in screw cap standard chromatographic glass vials, at a nominal resolution of $4 \mathrm{~cm}^{-1}$ accumulating 60 scans per spectra and using a laser power of $150 \mathrm{~mW}$.

\section{COMPUTATIONAL METHOD}

Density functional theory (DFT) calculations were carried out using the Gaussian 98 programme package [12]. The B3LYP functional was used with the 6-31 G(d,p) basis set. The calculations were performed using an isolated molecule of pilocarpine cation: $\mathrm{C}_{11} \mathrm{H}_{17} \mathrm{~N}_{2} \mathrm{O}_{2}^{+}$. The structure obtained from the $\mathrm{X}$-ray analyses of pilocarpine hydrochloride at $77 \mathrm{~K}$ was used as starting structure [10]. This structure was optimized and the vibrational wavenumbers were then calculated. The output file contained the optimized structure, the vibrational frequencies in the harmonic approximation, and the atomic displacements for each mode. At the optimized structure of the molecule, no imaginary frequency was obtained, proving that a true minimum of the potential energy surface was found. The calculated vibrational wave numbers were adjusted to compare with experimental Raman and IR frequencies.

\section{RESULTS AND DISCUSSION}

The crystal of pilocarpine hydrochloride at room temperature belongs to the monoclinic structure with $P 2_{1}\left(C_{2}^{2}\right)$ space group, with $\mathrm{Z}=2$, and lattice parameters $\mathrm{a}=11.057$ [10.965] $\AA, \mathrm{b}=9.212$ [9.177] $\AA, \mathrm{c}=6.697$ [6.507] $\AA$ and $\beta=110.05$ $[109.19]^{\circ}$ o (where the values in brackets hold for the $77 \mathrm{~K}$ determination) [10].

Figure 1 shows the molecular structure of the pilocarpine hydrochloride $\left(\mathrm{C}_{11} \mathrm{H}_{17} \mathrm{~N}_{2} \mathrm{O}_{2}^{+} \cdot \mathrm{Cl}^{-}\right)$. The numbering of the atoms in Fig. 1 follows that of Codding [10] in which the $N$-methylated nitrogens $\left(\mathrm{N}_{1}\right)$ are separated from the ether oxigen by four carbon atoms and from the carbonyl oxygen by five atoms. This labelling will be used in describing the parameters for optimized structure and the molecular wave vectors. The distribution of the two molecules of pilocarpine hydrochloride in the unit cell is showed in Fig. 2.

Tables 1, 2 and 3 show respectively, bond distances, bond angles and some selected torsion angles for pilocarpine cation, $\mathrm{C}_{11} \mathrm{H}_{17} \mathrm{~N}_{2} \mathrm{O}_{2}^{+}$, for optimized structure of the molecule (Cal) and that obtained from X-ray analysis (Exp) [10]. The results show that optimized structure was observed to reproduce the experiments with good agreement.

FT-Raman spectrum and the FT-infrared (IR) spectrum of pilocarpine hydrocholride $\mathrm{C}_{11} \mathrm{H}_{17} \mathrm{~N}_{2} \mathrm{O}_{2}^{+} . \mathrm{Cl}^{-}$are presented in Figs. 3(a) and 3(b), respectively.

The molecule of pilocarpine hydrochloride has $\mathrm{C}_{1}$ site symmetry, and therefore, 93 molecular vibrations among all 99 are allowed in the Raman spectrum. The couplings of vibrations due to the presence of two molecules in the unit cell give rise to twice greater number of modes in the crystal. The number of normal modes expected for the crystal is then, 198, distributed into irreducible representations of $\mathrm{C}_{2}$ factor group as $99(\mathrm{~A}+\mathrm{B})$; from these modes $99 \mathrm{~A}+99 \mathrm{~B}$ modes are Raman active. Assuming that the weakness of the intermolecular coupling causes negligible factor group splitting, the task is

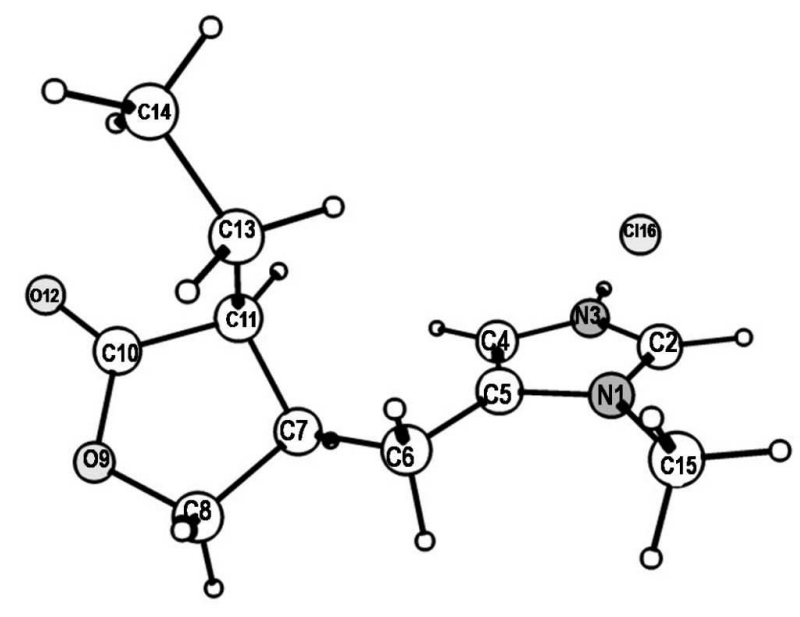

FIG. 1: The molecular structure of an isolated molecule of pilocarpine hydrochoride: $\mathrm{C}_{11} \mathrm{H}_{17} \mathrm{~N}_{2} \mathrm{O}_{2}^{+} \mathrm{Cl}^{-}$.

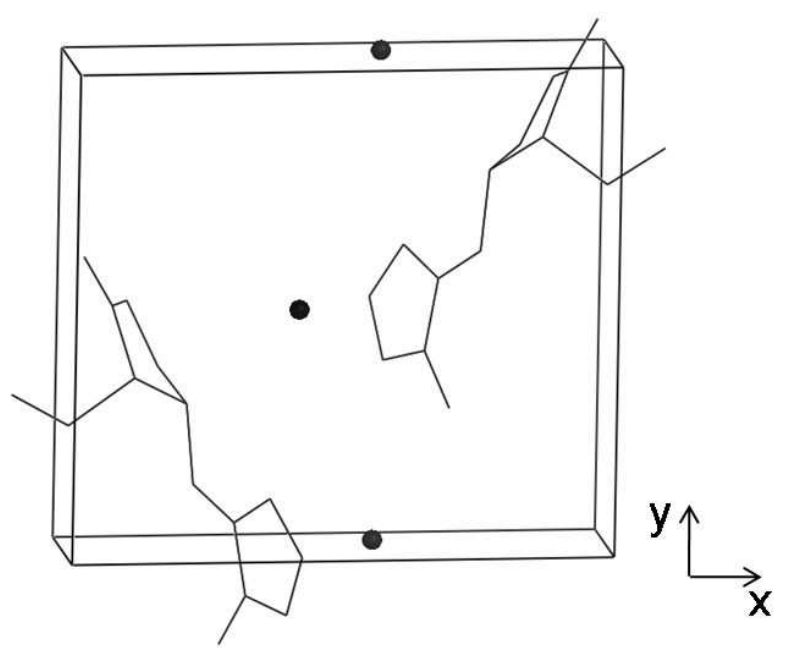

FIG. 2: Unit cell of pilocarpine hydrochloride; the $\mathrm{Z}$ plane is perpendicular to the figure.

simplified to the assignment of the 93 molecular modes. Table 4 lists a detailed description of assignments for vibrational wavenumbers of pilocarpine hydrochloride. In the first column the calculated values for the wavenumbers are given. We also present the experimental wavenumber values for the crystal obtained by FT-Raman and FT-IR spectroscopies (the second and third columns, respectively); the fourth column in Table 4 gives the assignment of the bands. In order to better visualise the vibrations, we refer to the two rings in the assignments of Table 4, as follows: imidazole ring type or 1-methylimidazole as $\mathbf{R} 1$ and $\gamma$ - lactone ring type or $\gamma$-butyrolactone as $\mathbf{R 2}$. The nomenclature employed in the classification of normal modes is given below the Table 4 .

The assignment for pilocarpine hydrochloride shows that most of the bands observed through FT-Raman and FT-IR spectroscopies correspond to a mixture of vibrational modes. The mixture of modes is common in molecules of $\mathrm{C}_{1}$ site sym- 
TABLE 1: Bond distances $(\AA)$ for Pilocarpine cation: $\mathrm{C}_{11} \mathrm{H}_{17} \mathrm{~N}_{2} \mathrm{O}_{2}^{+}$.

\begin{tabular}{ccc}
\hline & Exp. & Calc. \\
\hline $\mathrm{N}(1)-\mathrm{C}(2)$ & 1.328 & 1.337 \\
$\mathrm{~N}(1)-\mathrm{C}(5)$ & 1.384 & 1.399 \\
$\mathrm{~N}(1)-\mathrm{C}(15)$ & 1.459 & 1.469 \\
$\mathrm{C}(2)-\mathrm{N}(3)$ & 1.327 & 1.335 \\
$\mathrm{~N}(3)-\mathrm{C}(4)$ & 1.383 & 1.381 \\
$\mathrm{C}(4)-\mathrm{C}(5)$ & 1.362 & 1.369 \\
$\mathrm{C}(5)-\mathrm{C}(6)$ & 1.488 & 1.498 \\
$\mathrm{C}(6)-\mathrm{C}(7)$ & 1.523 & 1.535 \\
$\mathrm{C}(7)-\mathrm{C}(8)$ & 1.530 & 1.543 \\
$\mathrm{C}(7)-\mathrm{C}(11)$ & 1.544 & 1.549 \\
$\mathrm{C}(8)-\mathrm{O}(9)$ & 1.446 & 1.431 \\
$\mathrm{O}(9)-\mathrm{C}(10)$ & 1.354 & 1.368 \\
$\mathrm{C}(10)-\mathrm{C}(11)$ & 1.513 & 1.537 \\
$\mathrm{C}(10)-\mathrm{O}(12)$ & 1.206 & 1.200 \\
$\mathrm{C}(11)-\mathrm{C}(13)$ & 1.537 & 1.548 \\
$\mathrm{C}(13) \mathrm{C}(14)$ & 1.526 & 1.533 \\
\hline
\end{tabular}

TABLE 2: Bond angles $\left(^{\circ}\right)$ for Pilocarpine cation: $\mathrm{C}_{11} \mathrm{H}_{17} \mathrm{~N}_{2} \mathrm{O}_{2}^{+}$.

\begin{tabular}{lll}
\hline & Exp. & Calc. \\
\hline $\mathrm{C}(2)-\mathrm{N}(1)-\mathrm{C}(5)$ & 109.3 & 109.4 \\
$\mathrm{C}(2)-\mathrm{N}(1)-\mathrm{C}(15)$ & 124.7 & 124.9 \\
$\mathrm{C}(5)-\mathrm{N}(1)-\mathrm{C}(15)$ & 126.0 & 125.7 \\
$\mathrm{~N}(1)-\mathrm{C}(2)-\mathrm{N}(3)$ & 108.5 & 107.9 \\
$\mathrm{C}(2)-\mathrm{N}(3)-\mathrm{C}(4)$ & 108.9 & 109.5 \\
$\mathrm{~N}(3)-\mathrm{C}(4)-\mathrm{C}(5)$ & 107.0 & 107.2 \\
$\mathrm{~N}(1)-\mathrm{C}(5)-\mathrm{C}(4)$ & 106.3 & 105.9 \\
$\mathrm{~N}(1)-\mathrm{C}(5)-\mathrm{C}(6)$ & 121.8 & 122.5 \\
$\mathrm{C}(4)-\mathrm{C}(5)-\mathrm{C}(6)$ & 131.7 & 131.6 \\
$\mathrm{C}(5)-\mathrm{C}(6)-\mathrm{C}(7)$ & 113.4 & 114.2 \\
$\mathrm{C}(6)-\mathrm{C}(7)-\mathrm{C}(8)$ & 110.0 & 111.9 \\
$\mathrm{C}(6)-\mathrm{C}(7)-\mathrm{C}(11)$ & 118.3 & 118.7 \\
$\mathrm{C}(8)-\mathrm{C}(7)-\mathrm{C}(11)$ & 102.3 & 101.9 \\
$\mathrm{C}(7)-\mathrm{C}(8)-\mathrm{O}(9)$ & 105.0 & 105.3 \\
$\mathrm{C}(8)-\mathrm{O}(9)-\mathrm{C}(10)$ & 110.2 & 111.0 \\
$\mathrm{O}(9)-\mathrm{C}(10)-\mathrm{C}(11)$ & 110.9 & 109.7 \\
$\mathrm{O}(9)-\mathrm{C}(10)-\mathrm{O}(12)$ & 120.4 & 122.5 \\
$\mathrm{C}(11)-\mathrm{C}(10)-\mathrm{O}(12)$ & 128.6 & 127.9 \\
$\mathrm{C}(7)-\mathrm{C}(11)-\mathrm{C}(10)$ & 101.6 & 101.4 \\
$\mathrm{C}(7)-\mathrm{C}(11)-\mathrm{C}(13)$ & 114.6 & 116.2 \\
$\mathrm{C}(10)-\mathrm{C}(11)-\mathrm{C}(13)$ & 109.6 & 110.4 \\
$\mathrm{C}(11)-\mathrm{C}(13)-\mathrm{C}(14)$ & 114.7 & 113.5 \\
\hline
\end{tabular}

metry. The superposition of modes precludes a direct identification of the bands. However, an effort was carried out through this work to make a detailed description of assignments of vibrational modes of the crystal. In order to illustrate the assignment, atomic displacements corresponding to selected normal modes from the isolated molecular structure of pilocarpine hydrochloride are shown in Fig. 4.

Now we discuss the main calculated and observed vibrations of pilocarpine hydrochloride. Two fundamental units of the pilocarpine molecule are the rings. Imidazole ring, which is a characteristic part of pilocarpine, is present in several substances of biological interest, as for example, L-histidine amino acid [13-15], and in other substances [16,17]. Their vibrations spread over a large spectral range of wavenumbers. Lactone, the other ring, is also found in several different substances of biological interest [18-20]; for some of them spectroscopic studies have revealed the wavenumber of the main vibrations [19]. Calculations show that at low wavenumber $\left(\omega<150 \mathrm{~cm}^{-1}\right)$ where it is expected to be observed bands associated to lattice vibrations, some internal modes are also present. For example, torsional vibrations of the two rings are observed together with lattice modes at very low wavenumber. This should be expected because the rings are very large structures; so, we assign the bands in this spectral region as a mixture of lattice modes and torsional vibrations of the rings R1 and R2. Fig. 4(a) shows atomic displacements associated to deformations $\left\{\gamma_{\text {oop }}(\mathrm{R} 1), \delta_{\text {oop }}(\mathrm{R} 2)\right.$ [ $\tau(\mathrm{C} 10 \mathrm{O} 9 \mathrm{O} 12)], \mathrm{r}\left(\mathrm{C} 13 \mathrm{H}_{2}\right), \mathrm{r}\left(\mathrm{C}_{\left.14 \mathrm{H}_{3}\right)}\right\}$ corresponding to the strong Raman bands observed at $96 \mathrm{~cm}^{-1}\left(\omega_{\text {cal }}=97 \mathrm{~cm}^{-1}\right)$.

Another class of vibrations is related to deformation of rings. In plane ring deformation vibration appears in a large spectral region $\left(690-1900 \mathrm{~cm}^{-1}\right)$ and out of plane ring deformation vibration appears for $546<\omega<1140 \mathrm{~cm}^{-1}$. However, most of them are mixed with other kind of vibrations such as rocking and bending of $\mathrm{CH}$, torsion of $\mathrm{CH}_{2}$ and stretching of CC. Fig. 4(b) represents the mixtures of vibrational modes $\left\{\delta_{i p}(\mathrm{R} 2)\left[\mathrm{sc}(\mathrm{C} 8 \mathrm{C} 7 \mathrm{C} 11) ; v_{s}(\mathrm{C} 11 \mathrm{C} 10 \mathrm{O} 9)\right], \mathrm{r}\left(\mathrm{C}_{2} \mathrm{H}_{2} ; \mathrm{C} 13 \mathrm{H}_{2}\right)\right.$, $\left.\mathrm{r}\left(\mathrm{C} 14 \mathrm{H}_{3}\right), \delta(\mathrm{C} 7 \mathrm{H})\right\}$ giving rise to the strong Raman peak ob- 
TABLE 3: Some selected torsion angles $\left(^{\circ}\right)$ for Pilocarpine cation: $\mathrm{C}_{11} \mathrm{H}_{17} \mathrm{~N}_{2} \mathrm{O}_{2}^{+}$.

\begin{tabular}{ccc}
\hline & Exp. & Calc. \\
\hline $\mathrm{C}(2)-\mathrm{N}(1)-\mathrm{C}(5)-\mathrm{C}(6)$ & 176.7 & 179.1 \\
$\mathrm{C}(3)-\mathrm{C}(4)-\mathrm{C}(5)-\mathrm{C}(6)$ & -176.3 & -178.9 \\
$\mathrm{~N}(1)-\mathrm{C}(5)-\mathrm{C}(6)-\mathrm{C}(7)$ & 179.8 & 177.2 \\
$\mathrm{C}(4)-\mathrm{C}(5)-\mathrm{C}(6)-\mathrm{C}(7)$ & -4.2 & -4.1 \\
$\mathrm{C}(5)-\mathrm{C}(6)-\mathrm{C}(7)-\mathrm{C}(8)$ & 168.4 & 171.6 \\
$\mathrm{C}(5)-\mathrm{C}(6)-\mathrm{C}(7)-\mathrm{C}(11)$ & -74.5 & -70.1 \\
$\mathrm{C}(6)-\mathrm{C}(7)-\mathrm{C}(8)-\mathrm{O}(9)$ & 157.0 & 159.2 \\
$\mathrm{C}(6)-\mathrm{C}(7)-\mathrm{C}(11)-\mathrm{C}(10)$ & -149.7 & -153.3 \\
$\mathrm{C}(6)-\mathrm{C}(7)-\mathrm{C}(11)-\mathrm{C}(13)$ & -31.6 & -33.5 \\
$\mathrm{C}(7)-\mathrm{C}(8)-\mathrm{O}(9)-\mathrm{C}(10)$ & -20.4 & -20.2 \\
$\mathrm{O}(9)-\mathrm{C}(10)-\mathrm{C}(11)-\mathrm{C}(7)$ & 18.2 & 19.8 \\
$\mathrm{O}(12)-\mathrm{C}(10)-\mathrm{C}(11)-\mathrm{C}(7)$ & -162.8 & -161.2 \\
$\mathrm{C}(7)-\mathrm{C}(11)-\mathrm{C}(13)-\mathrm{C}(14)$ & 171.4 & 179.4 \\
\hline
\end{tabular}
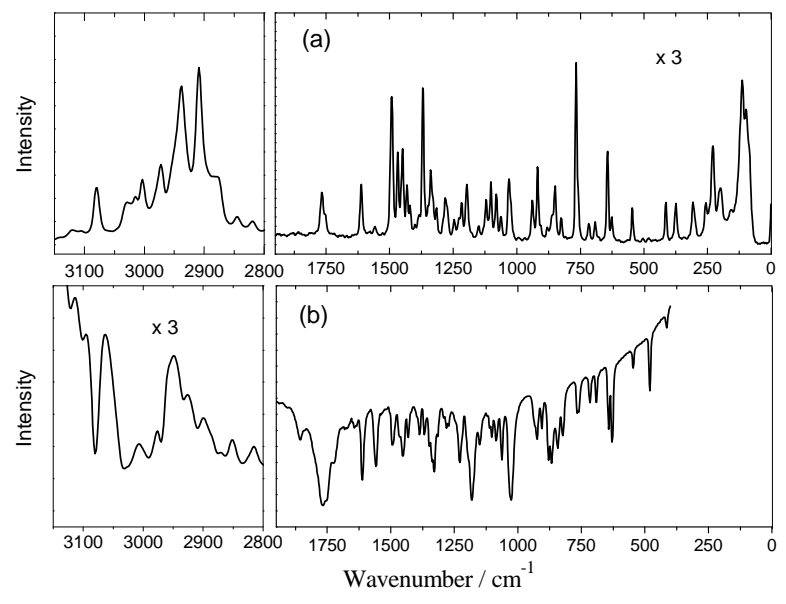

FIG. 3: (a) Pilocarpine hydrochloride FT-Raman spectrum. (b) Pilocarpine hydrochloride FT-IR spectrum.
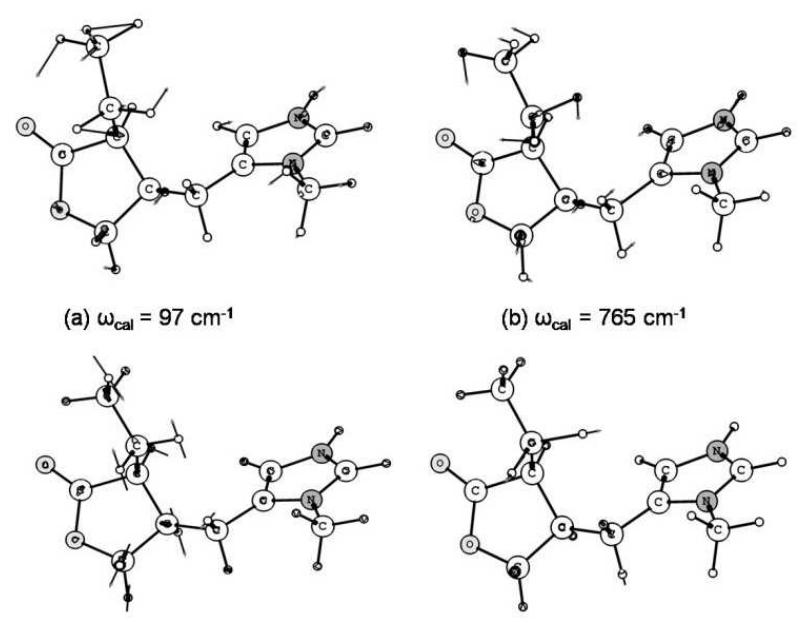

(c) $\omega_{\text {cal }}=1026 \mathrm{~cm}^{-1}$

(d) $\omega_{\text {cal }}=3024 \mathrm{~cm}^{-1}$

FIG. 4: Some selected representations of atomic vibrations corresponding to the strongest Raman bands of pilocarpine cation: $\mathrm{C}_{11}$ $\mathrm{H}_{17} \mathrm{~N}_{2} \mathrm{O}_{2}^{+}$. served at $766 \mathrm{~cm}^{-1}\left(\omega_{\text {cal. }}=765 \mathrm{~cm}^{-1}\right)$.

The whole structure presents deformation vibrations $\delta$ (all structure) at $\sim 650 \mathrm{~cm}^{-1}$ as well as at $\sim 1080 \mathrm{~cm}^{-1}$ and at $1300-1340 \mathrm{~cm}^{-1}$. A calculated $\delta$ (all structure) vibrational is also expected at $770 \mathrm{~cm}^{-1}$ but, possibly, is mixed with the $765 \mathrm{~cm}^{-1}$ complex vibration. Four strong IR bands are associated to ring deformation. Fig. 4(c) illustrates one of them observed at $1027 \mathrm{~cm}^{-1}\left(\omega_{\text {cal }}=1026 \mathrm{~cm}^{-1}\right)$, corresponding to $\left\{\delta_{\text {oop }}(\mathrm{R} 2)\left[\mathrm{v}_{\text {as }}(\mathrm{C} 11 \mathrm{C} 13 \mathrm{C} 14)\right]\right.$, wag $\left(\mathrm{C}^{3} \mathrm{H}_{2}\right), \mathrm{r}\left(\mathrm{C}^{2} \mathrm{H}_{2}\right.$;

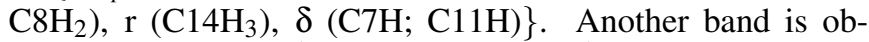
served at $1181 \mathrm{~cm}^{-1}\left(\omega_{\text {cal }}=1185 \mathrm{~cm}^{-1}\right)$, corresponding to

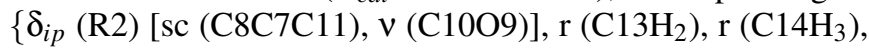
wag $\left.\left(\mathrm{C}^{2} \mathrm{H}_{2} ; \mathrm{C} \mathrm{H}_{2}\right), \delta(\mathrm{C} 7 \mathrm{H} ; \mathrm{C} 11 \mathrm{H})\right\}$. The other two strong IR bands are associated with the deformations $\left\{\delta_{i p}(\mathrm{R} 1)[\mathrm{V}\right.$

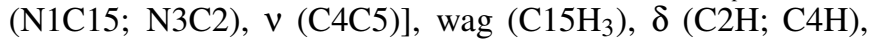
$\delta(\mathrm{N} 3 \mathrm{H})\}$ and $\left\{\delta_{i p}(\mathrm{R} 1)\right.$ [ $\left.\mathrm{v}(\mathrm{C} 4 \mathrm{C} 5 ; \mathrm{C} 5 \mathrm{C} 6), \mathrm{v}(\mathrm{C} 2 \mathrm{~N} 1)\right]$, wag $\left.\left(\mathrm{C} \mathrm{H}_{2}\right), \mathrm{r}\left(\mathrm{C} 15 \mathrm{H}_{3}\right), \delta(\mathrm{C} 2 \mathrm{H} ; \mathrm{C} 4 \mathrm{H}), \delta(\mathrm{N} 3 \mathrm{H})\right\}$, corresponding to the peaks observed at $1752 \mathrm{~cm}^{-1}\left(\omega_{\text {cal }}=1598 \mathrm{~cm}^{-1}\right)$ and $1767 \mathrm{~cm}^{-1}\left(\omega_{\text {cal }}=1655 \mathrm{~cm}^{-1}\right)$, respectively.

It is also interesting to note that the rocking vibrations of the three $\mathrm{CH}_{2}$ units $\left(\mathrm{C}_{6} \mathrm{H}_{2}, \mathrm{C}_{2} \mathrm{H}_{2}\right.$ and $\left.\mathrm{C} 13 \mathrm{H}_{2}\right)$ are observed at similar wavenumbers. On the contrary, although $\mathrm{CO}_{2}$ vibrations can be expected for pilocarpine molecule, rocking vibration of $\mathrm{CO}_{2}$ (a well characteristic vibration in amino acid crystal at $\left.500-540 \mathrm{~cm}^{-1}\right)$ is absent. This is because the only $\mathrm{CO}_{2}$ possibility is $\mathrm{C} 1009012$, but $\mathrm{O} 9$ is held at lactone ring; as a consequence, it is impossible to have a rocking $\mathrm{C} 1009012$ vibration.

Because there are many $\mathrm{C}-\mathrm{C}$ bonds in pilocarpine molecules the $\mathrm{CC}$ stretching vibrations are observed in a large range of wavenumbers. The lowest wavenumber value is for C11-C13 stretching, which was observed at $716 \mathrm{~cm}^{-1}$ while the highest wavenumber value corresponding to a $v(C C)$ is calculated at $1140 \mathrm{~cm}^{-1} \cdot \mathrm{v}(\mathrm{NC})$ vibrations contributes with bands observed at 939 and $1754 \mathrm{~cm}^{-1}$.

It is possible to note a marked localization of the scissoring vibrations $\left(\mathrm{CH}_{2}\right.$ and $\left.\mathrm{CH}_{3}\right)$ in the range $1382 \mathrm{~cm}^{-1}<\omega_{\text {cal }}<$ $1537 \mathrm{~cm}^{-1}$. As an example, the Raman band observed at 1492 $\mathrm{cm}^{-1}\left(\omega_{\text {cal }}=1493 \mathrm{~cm}^{-1}\right)$ corresponds to the scissoring vibration $\mathrm{sc}\left(\mathrm{C} 15 \mathrm{H}_{3}\right)$.

A large number of bands associated with overtones and combination tones may be found in the region about 2800 $\mathrm{cm}^{-1}$ due to the large number of bands in the region between $84 \mathrm{~cm}^{-1}$ and $1800 \mathrm{~cm}^{-1}$. The bands 2819, 2834 and 2844 
TABLE 4: Calculed vibrational wavenumbers unscaled, Raman band positions in units $\mathrm{of}^{-1}$ and assignments for vibrational modes of pilocarpine hydrochloride cation: $\mathrm{C}_{11} \mathrm{H}_{17} \mathrm{~N}_{2} \mathrm{O}_{2}^{+}$.

\begin{tabular}{|c|c|c|c|}
\hline$\overline{\omega_{\text {calc }}}$ & $\omega_{F T-R a m a n}$ & $\omega_{F T-I R}$ & Assignment \\
\hline 29 & & & $\gamma_{o o p}(\mathrm{R} 1), \mathrm{r}(\mathrm{C} 11 \mathrm{C} 13 \mathrm{C} 14)$ \\
\hline 43 & & & $\gamma_{\text {oop }}(\mathrm{R} 1), \gamma_{\text {oop }}(\mathrm{R} 2)$ \\
\hline 64 & $84 \mathrm{~m}$ & & $\gamma_{\text {oop }}(\mathrm{R} 1), \gamma_{\text {oop }}(\mathrm{R} 2)$ \\
\hline 97 & $96 \mathrm{~s}$ & & $\gamma_{\text {oop }}(\mathrm{R} 1), \delta_{\text {oop }}(\mathrm{R} 2)[\tau(\mathrm{C} 10 \mathrm{O} 9 \mathrm{O} 12)], \mathrm{r}\left(\mathrm{C} 13 \mathrm{H}_{2}\right), \mathrm{r}\left(\mathrm{C} 14 \mathrm{H}_{3}\right)$ \\
\hline 123 & $114 \mathrm{~s}$ & & $\gamma_{i p}(\mathrm{R} 1), \delta_{o o p}(\mathrm{R} 2)[\tau(\mathrm{C} 10 \mathrm{O} 9 \mathrm{O} 12)], \mathrm{r}\left(\mathrm{C} 13 \mathrm{H}_{2}\right), \mathrm{r}\left(\mathrm{C} 14 \mathrm{H}_{3}\right)$ \\
\hline 132 & & & $\tau\left(\mathrm{C}_{15} \mathrm{H}_{3}\right)$ \\
\hline 177 & $159 \mathrm{w}$ & & $\gamma_{o o p}(\mathrm{R} 1), \mathrm{r}(\mathrm{C} 11 \mathrm{C} 13 \mathrm{C} 14)$ \\
\hline 192 & $199 \mathrm{w}$ & & $\gamma_{\text {oop }}(\mathrm{R} 1), \delta_{\text {oop }}(\mathrm{R} 2)[\delta(\mathrm{C} 8 \mathrm{O} 9)], \mathrm{r}\left(\mathrm{C} 8 \mathrm{H}_{2}\right), \mathrm{r}\left(\mathrm{C} 14 \mathrm{H}_{3}\right)$ \\
\hline 212 & & & $\gamma_{\text {oop }}(\mathrm{R} 1), \gamma_{\text {oop }}(\mathrm{R} 2), \mathrm{r}(\mathrm{C} 11 \mathrm{C} 13 \mathrm{C} 14), \delta(\mathrm{C} 15 \mathrm{~N} 1)$ \\
\hline 214 & & & $\gamma_{o o p}(\mathrm{R} 1), \delta_{o o p}(\mathrm{R} 2)[\delta(\mathrm{C} 10 \mathrm{O} 12)], \mathrm{r}\left(\mathrm{C}^{2} \mathrm{H}_{2}\right), \mathrm{r}\left(\mathrm{C}_{14 \mathrm{H}_{3}} ; \mathrm{C} 15 \mathrm{H}_{3}\right)$ \\
\hline 228 & $229 \mathrm{~m}$ & & $\gamma_{\text {oop }}(\mathrm{R} 1), \delta_{\text {oop }}(\mathrm{N} 1 \mathrm{C} 15), \mathrm{r}\left(\mathrm{C} \mathrm{H}_{2}\right), \mathrm{r}\left(\mathrm{C} 14 \mathrm{H}_{3}\right)$ \\
\hline 247 & $256 \mathrm{w}$ & & $\gamma_{\text {oop }}(\mathrm{R} 1), \gamma_{\text {ip }}(\mathrm{R} 2), \delta_{\text {oop }}(\mathrm{N} 1 \mathrm{C} 15), \mathrm{r}\left(\mathrm{C} \mathrm{H}_{2}\right), \mathrm{r}\left(\mathrm{C} 14 \mathrm{H}_{3}\right)$ \\
\hline 288 & & & $\gamma_{\text {oop }}(\mathrm{R} 1), \delta_{\text {oop }}(\mathrm{N} 1 \mathrm{C} 15), \mathrm{r}\left(\mathrm{C} \mathrm{H}_{2} ; \mathrm{C} \mathrm{H}_{2} ; \mathrm{C}_{\left.13 \mathrm{H}_{2}\right)} ; \mathrm{r}\left(\mathrm{C} 14 \mathrm{H}_{3}\right)\right.$ \\
\hline 298 & $306 \mathrm{w}$ & & 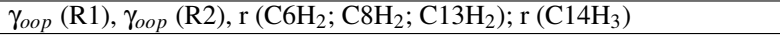 \\
\hline 366 & $374 \mathrm{w}$ & & sc $(\mathrm{C} 11 \mathrm{C} 13 \mathrm{C} 14), \gamma_{o o p}(\mathrm{R} 2), \mathrm{r}\left(\mathrm{C} \mathrm{H}_{2}\right)$ \\
\hline 412 & $414 \mathrm{w}$ & $415 \mathrm{vw}$ & $\gamma_{i p}(\mathrm{R} 1), \delta_{i p}(\mathrm{~N} 1 \mathrm{C} 15), \delta_{o o p}(\mathrm{R} 2)$ \\
\hline 479 & & $480 \mathrm{~m}$ & $\delta$ (all structure) \\
\hline 548 & $546 \mathrm{w}$ & $546 \mathrm{w}$ & $\delta_{\text {oop }}(\mathrm{R} 2)[\mathrm{sc}(\mathrm{C} 6 \mathrm{C} 7 \mathrm{C} 11) ; \mathrm{sc}(\mathrm{O} 9 \mathrm{C} 10 \mathrm{O} 12)], \mathrm{r}\left(\mathrm{C}^{2} \mathrm{H}_{2} ; \mathrm{C} 8 \mathrm{H}_{2}\right)$ \\
\hline 621 & & & $\delta_{\text {oop }}(\mathrm{R} 1)[\delta(\mathrm{C} 4 \mathrm{~N} 3 \mathrm{C} 2)]$ \\
\hline 627 & $625 \mathrm{vw}$ & $628 \mathrm{~m}$ & $\delta$ (all structure) \\
\hline 641 & $643 \mathrm{~m}$ & $643 \mathrm{~m}$ & $\delta$ (all structure) \\
\hline 654 & & & $\begin{array}{l}\delta_{o o p}(\mathrm{R} 1)[\delta(\mathrm{C} 4 \mathrm{C} 5 \mathrm{~N} 1)], \delta_{o o p}(\mathrm{C} 2 \mathrm{H} ; \mathrm{C} 4 \mathrm{H}), \delta_{o o p}(\mathrm{~N} 3 \mathrm{H}), \tau\left(\mathrm{C} \mathrm{H}_{2}\right), \\
\mathrm{r}\left(\mathrm{C} 8 \mathrm{H}_{2} ; \mathrm{C} 13 \mathrm{H}_{2}\right), \mathrm{r}\left(\mathrm{C} 15 \mathrm{H}_{3}\right)\end{array}$ \\
\hline 685 & & & $\delta_{o o p}(\mathrm{~N} 3 \mathrm{H})$ \\
\hline$\overline{690}$ & $691 \mathrm{vw}$ & $690 w$ & $\begin{array}{l}\delta_{i p}(\mathrm{R} 2)\left[v_{s}(\mathrm{C} 8 \mathrm{O} 9 \mathrm{C} 10)\right], \mathrm{r}\left(\mathrm{C} \mathrm{H}_{2} ; \mathrm{C} 8 \mathrm{H}_{2} ; \mathrm{C} 13 \mathrm{H}_{2}\right), \\
\mathrm{r}\left(\mathrm{C} 14 \mathrm{H}_{3}\right)\end{array}$ \\
\hline 710 & $716 \mathrm{vw}$ & $716 \mathrm{w}$ & $\begin{array}{l}\delta_{o o p}(\mathrm{R} 2)\left[\delta_{o o p}(\mathrm{C} 10 \mathrm{O} 12)\right], v(\mathrm{C} 11 \mathrm{C} 13), \delta(\mathrm{C} 11 \mathrm{H}), \mathrm{r}\left(\mathrm{C} 13 \mathrm{H}_{2}\right), \\
\mathrm{r}\left(\mathrm{C} 14 \mathrm{H}_{3}\right)\end{array}$ \\
\hline 765 & $766 \mathrm{~s}$ & $762 w$ & $\begin{array}{l}\delta_{i p}(\mathrm{R} 2)\left[\mathrm{sc}(\mathrm{C} 8 \mathrm{C} 7 \mathrm{C} 11) ; v_{s}(\mathrm{C} 11 \mathrm{C} 10 \mathrm{O} 9)\right], \mathrm{r}\left(\mathrm{C} 8 \mathrm{H}_{2} ; \mathrm{C} 13 \mathrm{H}_{2}\right), \\
\mathrm{r}\left(\mathrm{C} 14 \mathrm{H}_{3}\right), \delta(\mathrm{C} 7 \mathrm{H})\end{array}$ \\
\hline 770 & & & $\delta$ (all structure) \\
\hline 787 & $824 \mathrm{vw}$ & $822 \mathrm{~m}$ & $\delta_{o o p}(\mathrm{R} 1)\left[\delta_{o o p}(\mathrm{C} 2 \mathrm{H} ; \mathrm{C} 4 \mathrm{H})\right], \mathrm{r}\left(\mathrm{C} 6 \mathrm{H}_{2}\right)$ \\
\hline 834 & $850 \mathrm{w}$ & $843 \mathrm{~m}$ & $\delta_{o o p}(\mathrm{C} 2 \mathrm{H} ; \mathrm{C} 4 \mathrm{H}), \delta_{o o p}(\mathrm{~N} 3 \mathrm{H})$ \\
\hline 852 & $861 \mathrm{vw}$ & $866 \mathrm{~m}$ & $\begin{array}{l}\delta_{\text {oop }}(\mathrm{R} 2)\left[\mathrm{n}(\mathrm{C} 11 \mathrm{C} 13) ; v_{s}(\mathrm{C} 8 \mathrm{C} 7 \mathrm{C} 11)\right], \tau\left(\mathrm{C}_{13} \mathrm{H}_{2}\right), \mathrm{r}\left(\mathrm{C} 14 \mathrm{H}_{3}\right), \\
\delta(\mathrm{C} 7 \mathrm{H} ; \mathrm{C} 11 \mathrm{H})\end{array}$ \\
\hline 880 & & $880 \mathrm{~m}$ & $\begin{array}{l}\delta_{i p}(\mathrm{R} 2)\left[\delta(\mathrm{C} 10 \mathrm{O} 12) ; v_{s}(\mathrm{C} 11 \mathrm{C} 10 \mathrm{O} 9)\right], \tau\left(\mathrm{C}^{2} \mathrm{H}_{2} ; \mathrm{C}_{13} \mathrm{H}_{2}\right), \mathrm{r}\left(\mathrm{C} 14 \mathrm{H}_{3}\right), \\
\delta_{\text {oop }}(\mathrm{C} 4 \mathrm{H})\end{array}$ \\
\hline 917 & $918 \mathrm{~m}$ & $905 \mathrm{w}$ & $\delta_{o o p}(\mathrm{R} 2)\left[v_{s}(\mathrm{C} 11 \mathrm{C} 13 \mathrm{C} 14), v(\mathrm{C} 7 \mathrm{C} 8)\right], \delta_{o o p}(\mathrm{C} 4 \mathrm{H}), \delta(\mathrm{C} 7 \mathrm{H})$ \\
\hline 939 & $939 \mathrm{w}$ & $927 \mathrm{w}$ & $\delta_{i p}(\mathrm{R} 1)[v(\mathrm{C} 5 \mathrm{~N} 1), \mathrm{sc}(\mathrm{C} 2 \mathrm{~N} 3 \mathrm{C} 4)], \mathrm{r}\left(\mathrm{C} 15 \mathrm{H}_{3}\right)$ \\
\hline 950 & & & $\begin{array}{l}\delta_{\text {oop }}(\mathrm{R} 2)\left[\mathrm{v}_{s}(\mathrm{C} 11 \mathrm{C} 13 \mathrm{C} 14), v(\mathrm{C} 7 \mathrm{C} 8)\right], \delta(\mathrm{C} 7 \mathrm{H} ; \mathrm{C} 11 \mathrm{H}), \\
\mathrm{r}\left(\mathrm{C} 6 \mathrm{H}_{2} ; \mathrm{C} 8 \mathrm{H}_{2}\right), \mathrm{r}\left(\mathrm{C} 14 \mathrm{H}_{3}\right)\end{array}$ \\
\hline 1026 & $1031 \mathrm{~m}$ & $1027 \mathrm{~s}$ & $\begin{array}{l}\delta_{\text {oop }}(\mathrm{R} 2)\left[\mathrm{V}_{a s}(\mathrm{C} 11 \mathrm{C} 13 \mathrm{C} 14)\right], \text { wag }\left(\mathrm{C}_{13} \mathrm{H}_{2}\right), \mathrm{r}\left(\mathrm{C} \mathrm{H}_{2} ; \mathrm{C} 8 \mathrm{H}_{2}\right), \\
\mathrm{r}\left(\mathrm{C} 14 \mathrm{H}_{3}\right), \delta(\mathrm{C} 7 \mathrm{H} ; \mathrm{C} 11 \mathrm{H})\end{array}$ \\
\hline 1039 & & & $\begin{array}{l}\left.\delta_{i p}(\mathrm{R} 2)[v(\mathrm{C} 7 \mathrm{C} 8)], v_{a s} \mathrm{C} 11 \mathrm{C} 13 \mathrm{C} 14\right), \text { wag }\left(\mathrm{C} \mathrm{H}_{2}\right), \mathrm{r}\left(\mathrm{C} 8 \mathrm{H}_{2}\right), \\
\delta(\mathrm{C} 11 \mathrm{H})\end{array}$ \\
\hline 1068 & $1063 w$ & $1063 \mathrm{~m}$ & $\begin{array}{l}\delta_{\text {oop }}(\mathrm{R} 2)[\mathrm{v}(\mathrm{C} 7 \mathrm{C} 8 ; \mathrm{C} 10 \mathrm{O} 9)], \mathrm{r}\left(\mathrm{C}^{2} \mathrm{H}_{2} ; \mathrm{C}_{13} \mathrm{H}_{2}\right) ; \mathrm{r}\left(\mathrm{C} 14 \mathrm{H}_{3}\right), \\
\delta(\mathrm{C} 2 \mathrm{H} ; \mathrm{C} 4 \mathrm{H} ; \mathrm{C} 11 \mathrm{H}), \delta(\mathrm{N} 3 \mathrm{H})\end{array}$ \\
\hline 1078 & & & $\delta$ (all structure) \\
\hline 1083 & $1081 \mathrm{w}$ & $1085 \mathrm{w}$ & $\delta$ (all structure) \\
\hline 1106 & $1102 w$ & $1103 w$ & $\begin{array}{l}\delta_{o o p}(\mathrm{R} 2)[\mathrm{r}(\mathrm{C} 7 \mathrm{C} 11 \mathrm{C} 13) ; \nu(\mathrm{C} 8 \mathrm{O} 9 ; \mathrm{C} 10 \mathrm{O} 9)], v(\mathrm{C} 13 \mathrm{C} 14), \\
\mathrm{r}\left(\mathrm{C} 14 \mathrm{H}_{3}\right), \tau\left(\mathrm{C} 13 \mathrm{H}_{2}\right), \delta(\mathrm{C} 7 \mathrm{H} ; \mathrm{C} 11 \mathrm{H})\end{array}$ \\
\hline 1124 & $1121 \mathrm{w}$ & & $\delta_{i p}(\mathrm{R} 1)[v(\mathrm{C} 4 \mathrm{~N} 3)], \mathrm{r}\left(\mathrm{C} 15 \mathrm{H}_{3}\right), v(\mathrm{C} 7 \mathrm{C} 11), \delta(\mathrm{C} 2 \mathrm{H} ; \mathrm{C} 4 \mathrm{H})$ \\
\hline 1126 & & & $\begin{array}{l}\delta_{i p}(\mathrm{R} 1)[v(\mathrm{C} 5 \mathrm{~N} 1 ; \mathrm{C} 4 \mathrm{~N} 3)], \mathrm{r}\left(\mathrm{C} 14 \mathrm{H}_{3} ; \mathrm{C} 15 \mathrm{H}_{3}\right), \mathrm{r}\left(\mathrm{C} 8 \mathrm{H}_{2}\right), v(\mathrm{C} 7 \mathrm{C} 11) \\
\mathrm{d}(\mathrm{N} 3 \mathrm{H})\end{array}$ \\
\hline 1140 & & & $\begin{array}{l}\delta_{\text {oop }}(\mathrm{R} 2)[\mathrm{n}(\mathrm{C} 11 \mathrm{C} 13)], \mathrm{r}\left(\mathrm{C} 14 \mathrm{H}_{3} ; \mathrm{C}_{15} \mathrm{H}_{3}\right), \mathrm{r}\left(\mathrm{C} 8 \mathrm{H}_{2}\right), \delta(\mathrm{C} 2 \mathrm{H} ; \mathrm{C} 4 \mathrm{H}), \\
\delta(\mathrm{N} 3 \mathrm{H})\end{array}$ \\
\hline 1152 & $1151 \mathrm{vw}$ & $1150 \mathrm{w}$ & $\mathrm{r}\left(\mathrm{C} 15 \mathrm{H}_{3}\right), \delta(\mathrm{C} 2 \mathrm{H} ; \mathrm{C} 4 \mathrm{H}), \delta(\mathrm{N} 3 \mathrm{H})$ \\
\hline 1153 & & & $\delta$ (all structure) \\
\hline 1185 & & $1181 \mathrm{~s}$ & $\begin{array}{l}\delta_{i p}(\mathrm{R} 2)[\mathrm{sc}(\mathrm{C} 8 \mathrm{C} 7 \mathrm{C} 11), v(\mathrm{C} 10 \mathrm{O} 9)], \mathrm{r}\left(\mathrm{C} 13 \mathrm{H}_{2}\right), \mathrm{r}\left(\mathrm{C} 14 \mathrm{H}_{3}\right), \\
\text { wag }\left(\mathrm{C} 6 \mathrm{H}_{2} ; \mathrm{C} 8 \mathrm{H}_{2}\right), \delta(\mathrm{C} 7 \mathrm{H} ; \mathrm{C} 11 \mathrm{H})\end{array}$ \\
\hline 1207 & $1196 \mathrm{~m}$ & & $\begin{array}{l}\left.\delta_{i p}(\mathrm{R} 1) \text { [sc }(\mathrm{C} 5 \mathrm{C} 4 \mathrm{~N} 3)\right], \text { wag }\left(\mathrm{C} \mathrm{H}_{2}\right), \mathrm{r}\left(\mathrm{C} 8 \mathrm{H}_{2}\right), \\
\delta(\mathrm{C} 2 \mathrm{H} ; \mathrm{C} 4 \mathrm{H} ; \mathrm{C} 7 \mathrm{H} ; \mathrm{C} 11 \mathrm{H}), \delta(\mathrm{N} 3 \mathrm{H})\end{array}$ \\
\hline 1235 & $1218 \mathrm{w}$ & $1228 \mathrm{~m}$ & $\mathrm{r}\left(\mathrm{C} 14 \mathrm{H}_{3}\right), \tau\left(\mathrm{C}_{6} \mathrm{H}_{2} ; \mathrm{C} 8 \mathrm{H}_{2}\right), \delta(\mathrm{C} 2 \mathrm{H} ; \mathrm{C} 7 \mathrm{H} ; \mathrm{C} 11 \mathrm{H})$ \\
\hline 1253 & $1245 \mathrm{vw}$ & & $\mathrm{r}\left(\mathrm{C}_{14} \mathrm{H}_{3}\right), \tau\left(\mathrm{C}_{6} \mathrm{H}_{2} ; \mathrm{C} 8 \mathrm{H}_{2}\right), \delta(\mathrm{C} 4 \mathrm{H} ; \mathrm{C} 7 \mathrm{H} ; \mathrm{C} 11 \mathrm{H}), \delta(\mathrm{N} 3 \mathrm{H})$ \\
\hline 1267 & & $1272 \mathrm{vw}$ & $\begin{array}{l}\delta_{i p}(\mathrm{R} 1)[v(\mathrm{C} 4 \mathrm{~N} 3)], \mathrm{r}\left(\mathrm{C} 14 \mathrm{H}_{3}\right), \tau\left(\mathrm{C} 8 \mathrm{H}_{2} ; \mathrm{C}_{\left.13 \mathrm{H}_{2}\right)},\right. \\
\delta(\mathrm{C} 2 \mathrm{H} ; \mathrm{C} 4 \mathrm{H} ; \mathrm{C} 7 \mathrm{H} ; \mathrm{C} 11 \mathrm{H}), \delta(\mathrm{N} 3 \mathrm{H})\end{array}$ \\
\hline
\end{tabular}


Table 4 (Continued)

\begin{tabular}{|c|c|c|c|}
\hline$\overline{\omega_{\text {calc }}}$ & $\omega_{F T-\text { Raman }}$ & $\omega_{F T-I R}$ & Assignment \\
\hline 1280 & $1279 w$ & $1281 \mathrm{vw}$ & $\begin{array}{l}\delta_{i p}(\mathrm{R} 1)[v(\mathrm{C} 4 \mathrm{~N} 3)], \mathrm{r}\left(\mathrm{C} 14 \mathrm{H}_{3} ; \mathrm{C}_{15} \mathrm{H}_{3}\right), \tau\left(\mathrm{C} \mathrm{H}_{2} ; \mathrm{C} 3 \mathrm{H}_{2}\right), \\
\delta(\mathrm{C} 2 \mathrm{H} ; \mathrm{C} 4 \mathrm{H} ; \mathrm{C} 7 \mathrm{H} ; \mathrm{C} 11 \mathrm{H}), \delta(\mathrm{N} 3 \mathrm{H})\end{array}$ \\
\hline 1306 & & $1292 \mathrm{vw}$ & $\delta$ (all structure) \\
\hline 1310 & $1315 \mathrm{w}$ & & $\delta$ (all structure) \\
\hline 1339 & $1337 \mathrm{~m}$ & $1331 \mathrm{~m}$ & $\delta$ (all structure) \\
\hline 1349 & & & $\tau\left(\mathrm{C}_{6} \mathrm{H}_{2} ; \mathrm{C} 8 \mathrm{H}_{2} ; \mathrm{C} 13 \mathrm{H}_{2}\right), \delta(\mathrm{C} 11 \mathrm{H}), \mathrm{r}\left(\mathrm{C} 14 \mathrm{H}_{3}\right)$ \\
\hline 1371 & $1369 \mathrm{~s}$ & $1367 \mathrm{w}$ & $\begin{array}{l}\text { wag }\left(\mathrm{C} 14 \mathrm{H}_{3}\right), \text { wag }\left(\mathrm{C} 13 \mathrm{H}_{2}\right), \tau\left(\mathrm{C} 6 \mathrm{H}_{2} ; \mathrm{C} 8 \mathrm{H}_{2}\right), v_{a s}(\mathrm{C} 11 \mathrm{C} 13 \mathrm{C} 14), \\
\mathrm{r}(\mathrm{C} 5 \mathrm{C} 6 \mathrm{C} 7), \delta(\mathrm{C} 2 \mathrm{H} ; \mathrm{C} 7 \mathrm{H} ; \mathrm{C} 11 \mathrm{H}), \delta(\mathrm{N} 3 \mathrm{H})\end{array}$ \\
\hline 1382 & $1387 \mathrm{vw}$ & $1387 w$ & $\begin{array}{l}\text { sc }\left(\mathrm{C} 14 \mathrm{H}_{3}\right), \tau\left(\mathrm{C}_{6} \mathrm{H}_{2} ; \mathrm{C} 8 \mathrm{H}_{2}\right), \text { wag }\left(\mathrm{C}_{13} \mathrm{H}_{2}\right), v_{a s}(\mathrm{C} 11 \mathrm{C} 13 \mathrm{C} 14), \\
\delta(\mathrm{C} 7 \mathrm{H} ; \mathrm{C} 11 \mathrm{H})\end{array}$ \\
\hline 1392 & & & $\begin{array}{l}\delta_{i p}(\mathrm{R} 1)[\mathrm{sc}(\mathrm{C} 4 \mathrm{C} 5 \mathrm{~N} 3) ; v(\mathrm{~N} 1 \mathrm{C} 2 ; \mathrm{N} 1 \mathrm{C} 5)], \mathrm{sc}\left(\mathrm{C} 15 \mathrm{H}_{3}\right), \\
\text { wag }\left(\mathrm{C} 6 \mathrm{H}_{2} ; \mathrm{C} 8 \mathrm{H}_{2} ; \mathrm{C} 13 \mathrm{H}_{2}\right), v_{a s}(\mathrm{C} 5 \mathrm{C} 6 \mathrm{C} 7), \delta(\mathrm{C} 7 \mathrm{H})\end{array}$ \\
\hline 1408 & & & 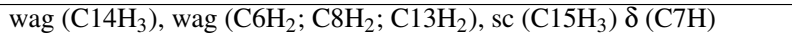 \\
\hline 1424 & $1422 \mathrm{vw}$ & & wag $\left(\mathrm{C} 14 \mathrm{H}_{3}\right)$, wag $\left(\mathrm{C}^{2} \mathrm{H}_{2} ; \mathrm{C} 8 \mathrm{H}_{2} ; \mathrm{C} 13 \mathrm{H}_{2}\right), \delta(\mathrm{C} 7 \mathrm{H} ; \mathrm{C} 11 \mathrm{H})$ \\
\hline 1438 & $1433 w$ & $1432 \mathrm{w}$ & wag $\left(\mathrm{C} 14 \mathrm{H}_{3}\right)$ \\
\hline 1468 & $1449 \mathrm{~m}$ & $1452 \mathrm{~m}$ & wag $\left(\mathrm{C} 15 \mathrm{H}_{3}\right), \mathrm{sc}\left(\mathrm{C}^{2} \mathrm{H}_{2}\right), \delta(\mathrm{C} 2 \mathrm{H}), \delta(\mathrm{N} 3 \mathrm{H})$ \\
\hline 1480 & $1468 \mathrm{~m}$ & & 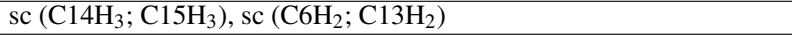 \\
\hline 1493 & $1492 \mathrm{~s}$ & $1492 \mathrm{w}$ & $\mathrm{sc}\left(\mathrm{C} 15 \mathrm{H}_{3}\right)$ \\
\hline 1506 & & & $\mathrm{sc}\left(\mathrm{C} 14 \mathrm{H}_{3} ; \mathrm{C} 15 \mathrm{H}_{3}\right), \mathrm{sc}\left(\mathrm{C}_{6} \mathrm{H}_{2} ; \mathrm{C} 13 \mathrm{H}_{2}\right)$ \\
\hline$\overline{1511}$ & & & $\mathrm{sc}\left(\mathrm{C} 14 \mathrm{H}_{3} ; \mathrm{C} 15 \mathrm{H}_{3}\right), \mathrm{sc}\left(\mathrm{C} 13 \mathrm{H}_{2}\right), \delta(\mathrm{N} 3 \mathrm{H})$ \\
\hline 1512 & & & 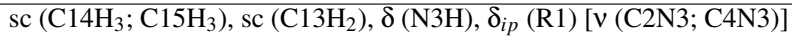 \\
\hline 1518 & & & $\mathrm{sc}\left(\mathrm{C} 14 \mathrm{H}_{3} ; \mathrm{C} 15 \mathrm{H}_{3}\right), \mathrm{sc}\left(\mathrm{C}_{6} \mathrm{H}_{2} ; \mathrm{C} 13 \mathrm{H}_{2}\right), \delta(\mathrm{N} 3 \mathrm{H})$ \\
\hline 1523 & $1559 \mathrm{vw}$ & $1558 \mathrm{~m}$ & $\mathrm{sc}\left(\mathrm{C} 14 \mathrm{H}_{3}\right), \mathrm{sc}\left(\mathrm{C} 13 \mathrm{H}_{2}\right)$ \\
\hline 1537 & $1611 \mathrm{w}$ & $1613 \mathrm{~m}$ & $\mathrm{sc}\left(\mathrm{C}_{2} \mathrm{H}_{2}\right)$ \\
\hline 1598 & $1754 \mathrm{vw}$ & $1752 \mathrm{~s}$ & $\begin{array}{l}\delta_{i p}(\mathrm{R} 1)[v(\mathrm{~N} 1 \mathrm{C} 15 ; \mathrm{N} 3 \mathrm{C} 2), v(\mathrm{C} 4 \mathrm{C} 5)], \text { wag }\left(\mathrm{C} 15 \mathrm{H}_{3}\right), \mathrm{d}(\mathrm{C} 2 \mathrm{H} ; \mathrm{C} 4 \mathrm{H}), \\
\delta(\mathrm{N} 3 \mathrm{H})\end{array}$ \\
\hline 1655 & $1767 w$ & $1767 \mathrm{~s}$ & $\begin{array}{l}\delta_{i p}(\mathrm{R} 1)[v(\mathrm{C} 4 \mathrm{C} 5 ; \mathrm{C} 5 \mathrm{C} 6), v(\mathrm{C} 2 \mathrm{~N} 1)], \text { wag }\left(\mathrm{C} \mathrm{H}_{2}\right), \mathrm{r}\left(\mathrm{C} 15 \mathrm{H}_{3}\right), \delta(\mathrm{C} 2 \mathrm{H} ; \\
\mathrm{C} 4 \mathrm{H}), \delta(\mathrm{N} 3 \mathrm{H})\end{array}$ \\
\hline \multirow[t]{4}{*}{1892} & & $1863 \mathrm{w}$ & $\delta_{i p}(\mathrm{R} 2)\left[v_{s}(\mathrm{C} 10=\mathrm{O} 12)\right]$ \\
\hline & $2819 \mathrm{w}$ & & combination \\
\hline & & $2834 \mathrm{vw}$ & combination \\
\hline & $2844 \mathrm{w}$ & & combination \\
\hline 3024 & $2908 \mathrm{vs}$ & $2911 \mathrm{vw}$ & $v_{s}\left(\mathrm{C}_{6} \mathrm{H}_{2} ; \mathrm{C} 13 \mathrm{H}_{2}\right), \mathrm{v}(\mathrm{C} 7 \mathrm{H})$ \\
\hline 3027 & $2938 \mathrm{vs}$ & $2935 \mathrm{vw}$ & $v_{s}\left(\mathrm{C}_{6} \mathrm{H}_{2} ; \mathrm{C} 8 \mathrm{H}_{2} ; \mathrm{C} 13 \mathrm{H}_{2}\right), v(\mathrm{C} 7 \mathrm{H})$ \\
\hline 3036 & $2972 \mathrm{~s}$ & $2970 \mathrm{w}$ & $v_{s}\left(\mathrm{C} \mathrm{H}_{2} ; \mathrm{C} 8 \mathrm{H}_{2} ; \mathrm{C} 13 \mathrm{H}_{2}\right), v(\mathrm{C} 7 \mathrm{H} ; \mathrm{C} 11 \mathrm{H})$ \\
\hline 3045 & $3004 \mathrm{~s}$ & $3007 \mathrm{w}$ & $v_{a s}\left(\mathrm{C} \mathrm{H}_{2}\right), v_{s}\left(\mathrm{C} 8 \mathrm{H}_{2}\right), v(\mathrm{C} 7 \mathrm{H} ; \mathrm{C} 11 \mathrm{H})$ \\
\hline 3055 & $3025 \mathrm{~m}$ & $3030 \mathrm{w}$ & $v_{s}\left(\mathrm{C} 14 \mathrm{H}_{3}\right), v_{a s}\left(\mathrm{C} 13 \mathrm{H}_{2}\right), v(\mathrm{C} 11 \mathrm{H})$ \\
\hline 3061 & & & $v_{s}\left(\mathrm{C} 14 \mathrm{H}_{3}\right), v_{a s}\left(\mathrm{C} 6 \mathrm{H}_{2} ; \mathrm{C} 13 \mathrm{H}_{2}\right), v_{s}\left(\mathrm{C} 8 \mathrm{H}_{2}\right), v(\mathrm{C} 11 \mathrm{H})$ \\
\hline 3077 & $3079 \mathrm{~s}$ & & $v_{s}\left(\mathrm{C} 15 \mathrm{H}_{3}\right), v_{a s}\left(\mathrm{C} \mathrm{H}_{2} ; \mathrm{C} 13 \mathrm{H}_{2}\right), v(\mathrm{C} 7 \mathrm{H} ; \mathrm{C} 11 \mathrm{H})$ \\
\hline 3084 & & & $v_{s}\left(\mathrm{C} 15 \mathrm{H}_{3}\right), \mathrm{v}_{a s}\left(\mathrm{C} 13 \mathrm{H}_{2}\right), \mathrm{v}(\mathrm{C} 7 \mathrm{H} ; \mathrm{C} 11 \mathrm{H})$ \\
\hline 3085 & & & $v_{s}\left(\mathrm{C}_{15 \mathrm{H}_{3}}\right), v_{a s}\left(\mathrm{C} \mathrm{H}_{2} ; \mathrm{C} 13 \mathrm{H}_{2}\right), v(\mathrm{C} 7 \mathrm{H} ; \mathrm{C} 11 \mathrm{H})$ \\
\hline 3124 & $3116 \mathrm{vw}$ & & $v_{a s}\left(\mathrm{C} 14 \mathrm{H}_{3}\right), v_{a s}\left(\mathrm{C} \mathrm{H}_{2} ; \mathrm{C} 13 \mathrm{H}_{2}\right), v(\mathrm{C} 7 \mathrm{H} ; \mathrm{C} 11 \mathrm{H})$ \\
\hline 3125 & & & $v_{a s}\left(\mathrm{C} 14 \mathrm{H}_{3}\right), v_{a s}\left(\mathrm{C} 8 \mathrm{H}_{2} ; \mathrm{C} 13 \mathrm{H}_{2}\right), v(\mathrm{C} 7 \mathrm{H} ; \mathrm{C} 11 \mathrm{H})$ \\
\hline 3157 & & & $\mathrm{v}_{a s}\left(\mathrm{C} 14 \mathrm{H}_{3}\right), \mathrm{v}_{a s}\left(\mathrm{C} 13 \mathrm{H}_{2}\right)$ \\
\hline 3170 & & & $v_{a s}\left(\mathrm{C}_{15} \mathrm{H}_{3}\right)$ \\
\hline 3188 & & & $v_{a s}\left(\mathrm{C}_{15} \mathrm{H}_{3}\right)$ \\
\hline
\end{tabular}

$\tau=$ twisting; $\gamma=$ torsion; sc= scissoring; wag = wagging; $\delta=$ deformation; $\delta_{i p}=$ deformation in plane; $\delta_{o o p}=$ deformation out of plane; $\mathrm{r}=$ rock; $\mathrm{v}=$ stretching; $\mathrm{v}_{a s}=$ asymmetric stretching; $\mathrm{v}_{s}=$ symmetric stretching. $\mathrm{vs}=$ very strong $; \mathrm{s}=$ strong; $\mathrm{m}=$ medium; $\mathrm{w}=$ weak; $\mathrm{vw}=$ very weak.

at least one strong Raman band. Thus, the modes at 96, 114, 766,1369 , and $1492 \mathrm{~cm}^{-1}$ can be involved.

The spectral region between 2800 and $3150 \mathrm{~cm}^{-1}$ of the Raman spectrum of pilocarpine hydrochloride crystal consists of a series of very intense Raman bands, and a series of less intense IR bands. However, all bands are well resolved, allowing for their identification as listed in Table 4. For organic crystals the region about $3000 \mathrm{~cm}^{-1}$, in general, contains the bands originated from $\mathrm{C}-\mathrm{H}, \mathrm{CH}_{2}, \mathrm{CH}_{3}$, and $\mathrm{N}-\mathrm{H}$ vibrations $[21,22]$. For some materials this region condenses very important informations, being a tool to understand conformation of the molecules in the unit cell or even interactions such as hydrogen bonds. For example, a study on L-methionine crystal have shown that the behaviour of Raman bands under pressure in this spectral region can be understood as consequence of structural changes instead of simple conformational changes of molecules in the unit cell [23]. So, the understand of the origin of these bands can be fundamental to understand the behaviour of pilocarpine hydrochloride under different conditions, in particular, related to the conditions found in drug artefacts. The scheme of Fig. 4(d) shows, as an example, the mixtures of stretching modes $\left\{\mathrm{v}_{s}\left(\mathrm{C}_{6} \mathrm{H}_{2} ; \mathrm{C} 13 \mathrm{H}_{2}\right), \mathrm{v}(\mathrm{C} 7 \mathrm{H})\right\}$ corresponding to a very strong Raman band observed at $2908 \mathrm{~cm}^{-1}\left(\omega_{\text {cal }}\right.$ $=3024 \mathrm{~cm}^{-1}$ ).

\section{CONCLUSIONS}

The phonon spectrum of the pilocarpine hydrochloride, a potential pharmaceutical substance to be used in several dis- 
ease treatments, was measured at room temperature through FT-Raman and FT-IR techniques. Density functional theory calculations were carried out by using the Gaussian 98 package and the B3LYP functional with the 6-31 G(d,p) basis set. The calculations were observed to reproduce the experiments with good agreement. This agreement allowed us the assignment of the observed wavenumbers to atomic motions in the molecules. In particular, it was observed that most bands are associated to mixing of vibrational modes, even in the low wavenumber region where, generally, the lattice modes are found. The absence of stretching vibrations of water molecule which can be observed at $\sim 3400 \mathrm{~cm}^{-1}$ in this region indicates that the crystal is free of water molecules.

\section{Acknowledgments}

We thank CENAPAD-SP for the use of the GAUSSIAN 98 software package and for computational facilities through the project reference "proj373". Financial support from CNPq, CAPES and FUNCAP is also acknowledged. One of us (AMRT) thanks Universidade Regional do Cariri for allowing him to spend one year at UFC to develop his pos-doc research.
[1] E. Urlaub, J. Popp, W. Kiefer, G. Bringmann, D. Koppler, H. Schneider, U. Zimmermann, and B. Schrader. Biospectrosc. 4, 113 (1998).

[2] R.J.H. Clark, R.E. Hester (eds). Spectroscopy of Biological Systems. Advances in Spectroscopy, vol. 13. Wiley: Chichester, 1986.

[3] W. Sneader. Drug Prototypes and their Exploitation. Wiley: Chichester, 1996.

[4] M. Andrade-Neto, E.R. Silveira, P.H. Mendes. Phytochem. 42, 885 (1996).

[5] B. Levy, R.P. Ahliquist. J. Pharmacol. Erp. Ther. 137, 219 (1962).

[6] P.G. Watson. Br. J. Ophthalmol. 56, 145 (1972).

[7] B.N. Schwartz. Engl. J. Med. 290, 182 (1978).

[8] L.S. Goodman, A. Gilman. The Pharmacological Basis of Therapeutics, 6th ed.; MacMillan: New York, 1980; p 97.

[9] S. Fregerslev, S.E. Rasmussen. Acta Chem. Scand. 22, 2541 (1968).

[10] P.W. Codding, M.N.G. James. Acta Crystallogr. B40, 42 (1984).

[11] J.M. Schulman, M.L. Sabio, R.L. Disch. J. Med. Chem. 26, 817 (1983).

[12] M.J. Frisch, G.W. Trucks, H.B. Schlegel, G.E. Scuseria, M.A. Robb, J.R. Cheeseman, V.G. Zakrzewski, J.A. Montgomery, R.E. Stratmann Jr, J.C. Burant, S. Dapprich, J.M. Millam, A.D. Daniels, K.N. Kudin, M.C. Strain, O. Farkas, J. Tomasi, V. Barone, M. Cossi, R. Cammi, B. Mennucci, C. Pomelli, C. Adamo, S. Clifford, J. Ochterski, G.A. Petersson, P.Y. Ayala, Q. Cui, K. Morokuma, P. Salvador, J.J. Dannenberg, D.K. Malick, A.D. Rabuck, K. Raghavachari, J.B. Foresman, J. Cioslowski, J.V. Ortiz, A.G. Baboul, B.B. Stefanov, G. Liu, A. Liashenko, P. Piskorz, I. Komaromi, R. Gomperts, R.L. Martin, D.J. Fox, T.
Keith, M.A. Al-Laham, C.Y. Peng, A. Nanayakkara, M. Challacombe, P.M.W. Gill, B. Johnson, W. Chen, M.W. Wong, J.L. Andres, C. Gonzalez, M. Head-Gordon, E.S. Replogle, J.A. Pople. Gaussian 98 (Revision A.11.2). Gaussian: Pittsburgh, PA, 2001.

[13] M. Tasumi , I. Harada, T. Takamatsu, S. Takahashi. J. Raman Spectrosc. 12, 149 (1982).

[14] T. Miura, T. Satoh, A. Hori-i, H. Takeuchi. J. Raman Spectrosc. 29, 41 (1998).

[15] J.L.B. Faria, F.M. Almeida, O. Pilla, F. Rossi, J.M. Sasaki, F.E.A. Melo, J.M. Filho, P.T.C. Freire. J. Raman Spectrosc. 35, 242 (2004).

[16] A. Torreggiani, A. Degli Esposti, M. Tamba, G. Marconi, G. Fini , J. Raman Spectrosc. 37, 291 (2006).

[17] B.H. Loo, Y. Tse, K. Parsons, C. Adelman, A. El-Hage, Y.G. Lee, J. Raman Spectrosc. 37, 299 (2006).

[18] W. Zhang, K. Krohn, J. Ding, Z.H. Miao, X.H. Zhou, S.H. Chen, G. Pescitelli, P. Salvadori, T. Kurtan, Y.W. Guo. J. Nat. Prod. 71, 961 (2008).

[19] J. Binoy, J.P. Abraham, Joe I. Hybert, V. George, V.S. Jayakumar, J. Aubard, Nielsen O. Faurskov. J. Raman Spectrosc. 36, 63 (2005).

[20] S. Basu, Y. Gerchman, C.H. Collins, F.H. Arnold, R. Weiss, Nature 434, 1130 (2005).

[21] B.L. Silva, P.T.C. Freire, F.E.A. Melo, I. Guedes, M.A.A. Silva, J.M. Filho, A.J.D. Moreno. Braz. J. Phys. 28, 19 (1998).

[22] P.F. Façanha Filho, P.T.C. Freire, K.C.V. Lima, J.M. Filho, F.E.A. Melo, P.S. Pizani. Braz. J. Phys. 38, 131 (2008).

[23] J.A. Lima Jr., P.T.C. Freire, F.E.A. Melo, V. Lemos, J. MendesFilho, P.S. Pizani. J. Raman Spectrosc. 39, 1356 (2008). 